\title{
Urban mobility and resilience: exploring Boston's urban mobility network through twitter data
}

Sahar Mirzaee ${ }^{1 *}$ (D) and Qi Wang ${ }^{2}$

\author{
* Correspondence: s.mirzaee@ \\ northeastern.edu \\ 'Department of Public Policy and \\ Urban Affairs, Northeastern \\ University, Boston, MA 02115, USA \\ Full list of author information is \\ available at the end of the article
}

\begin{abstract}
Human mobility connects urban dwellers and neighborhoods and impacts social equity. An in-depth understanding of human mobility helps to enhance urban resilience. However, limited research has focused on mobility resilience. Building on previous research, this study looks at the neighborhood connectivity enabled by urban mobility. We analyze the aggregated mobility patterns in Boston through the coupling of network structure and social characteristics. Geocoded twitter data combined with socioeconomic datasets were used to create a mobility-based urban network. Through the quantitative analysis, we found that the social segregation in Boston shapes its mobility network. Network communities identified by the Louvain modularity algorithm are often self-containing, meaning that their residents are more likely to move within their communities. A multinomial regression reveals that spatial racial and income segregation has a strong impact on the dynamic segregation of the network. The beneficial network characteristics -e.g. higher density and wellconnected motifs- are less present in areas with bolder presence of minorities. Thus, the resilience state is not equitable among neighborhoods of different income levels and races, indicating that the resilience measures of urban networks need to be adapted according to sociodemographic characteristics.
\end{abstract}

Keywords: Urban mobility, Resilience, Urban systems, Network science, Social segregation, Network motifs

\section{Introduction}

Cities are hybrid systems consisting of entangled entities and thus should be treated as one adaptive complex system (Amir and Kant 2018). Because of the high complexity, advancing urban resilience requires studying and engineering urban areas as a set of interactive social, environmental, structural, and governance systems. One of the biggest challenges of enhancing urban resilience is to identify processes that require changes in this context. It can only be achieved through case studies that are representative of the real world accompanied by descriptive and explanatory quantitative analyses that can also raise new questions (Hill et al. 2012).

(c) The Author(s). 2020 Open Access This article is licensed under a Creative Commons Attribution 4.0 International License, which permits use, sharing, adaptation, distribution and reproduction in any medium or format, as long as you give appropriate credit to the original author(s) and the source, provide a link to the Creative Commons licence, and indicate if changes were made. The images or other third party material in this article are included in the article's Creative Commons licence, unless indicated otherwise in a credit line to the material. If material is not included in the article's Creative Commons licence and your intended use is not permitted by statutory regulation or exceeds the permitted use, you will need to obtain permission directly from the copyright holder. To view a copy of this licence, visit http://creativecommons.org/licenses/by/4.0/. 
Mobility resilience is a critical aspect of urban resilience. Mobility, a basic human behavior, is the "the micro-geographies of everyday life" (Adey 2010). It lies within the intersection of many urban systems to generate and accumulate wealth, support business, and maintain engineering and social infrastructures. During manmade and natural disasters, maintaining mobility resilience could be a matter of life and death and help urban dwellers "remain resilient in the face of adversity" (United States 2017) and withstanding and recovering rapidly.

Despite the critical importance, human mobility research has long been focusing on the discovery of systematic behaviors within a certain range of the stable state, including the high uniformity (Jurdak et al. 2015; Brockmann et al. 2006), ultraslow diffusion (Toole et al. 2015; Song et al. 2010a, b; Noulas et al. 2012; Cuttone et al. 2018; Yan et al. 2014), periodicity (Cho et al. 2011; Liang et al. 2012), high predictability (Bagrow et al. 2011; Schneider et al. 2013a), and motif composition (Schneider et al. 2013a, b; Candia et al. 2008). Limited research has been conducted to understand how mobility enables connectivity between urban neighborhoods. Also, there is a dearth of studies that attempted to understand study how socio-demographics impact the mobility-based social networks.

Building on a comprehensive definition of urban resilience and through a case study of Boston, this study focuses on inter-neighborhood mobility structure and its relationship with socioeconomic characteristics of residents to better understand the weaknesses, strengths, and segregation patterns of the mobility network of the city. The rationale of the study is that humans are inevitably and continuously an integral part of many networks. Researchers have applied a variety of approaches to gather and process large quantities of data to systematically model the networks around us and capture the dynamic nature of real-world networks (Vespignani 2012). The diverse use of concepts, tools, and methods from network science in many fields and the interdependent nature of the systems of study, make it a remarkably suitable instrument to study mobility networks. Understanding the underlying characteristics of such network are vital in mitigating the negative impacts of hazards and improving urban resilience.

The rest of the article is organized in the following orders. In Section 2, we provide a brief review of research background in related areas. We discuss the importance of mobility for social resilience in urban areas and we review the literature on urban mobility and discuss the differences between urban mobility systems and urban transportation systems. The clarification of the differences serves as the foundation to discuss the differences in resilience studies of the two systems. The last part of the Section 2 explains motifs and their role in mobility network studies. In Section 3, we introduce both the data and the network analysis methods and measures used in this study. Then it is followed by Section 4 to present the results from our analysis. We present our discovery of the network structures and social characteristics of network communities hidden in the mobility network in Boston. Also, we discuss the motifs in these network communities. The paper ends with an acknowledge of certain limitations (Section 5) and our conclusions (Section 6).

\section{Background}

Urban resilience

Urban areas are a combination of ecological, technical, and social systems. Ecological or technological approaches to enhance resilience of urban areas will fail without the 
third social consideration (Grimm et al. 2008). Urban systems are not merely aggregation of people and technology, rather they are hybrid systems and entangled entities that should be looked at as an adaptive whole (Amir and Kant 2018). Although urban resilience is still a vague concept that often-times lacks operationalizability, recent definitions of urban resilience agree on the interdependency of urban systems (e.g. Coaffee 2008; Paton and Johnston 2001). In this study, we define urban resilience as:

the ability of an urban system, its social units (such as individuals, communities, institutions, governments, etc.), and its technical units (urban infrastructure) to recover from hazards while maintaining functional continuity of their substituents and as a whole, and mitigating negative impacts of future hazards through practice of resilience planning.

Building on this definition, this study focuses on social aspect of urban resilience and concentrates on affected neighborhoods and demographics in cases of disruption to better understand the weaknesses, strengths, and the level of functional continuity of mobility network of Boston. Understanding the underlying characteristics of mobility networks are vital in mitigating the negative impacts of hazards and improving urban resilience.

\section{Mobility and social resilience}

Urban areas consist of many interconnected and interdependent systems. These systems include the urban ecology, urban infrastructure, social networks, governance networks, and others. Enhancing urban resilience requires understanding these systems and improving upon them individually and collectively. The social aspect of urban resilience requires social integration of people with different races and income levels (Fonseca et al. 2019). Social integration stands at the opposite of social segregation; the former benefits social resilience and the latter hinders it.

Social segregation limits housing opportunities and has serious detrimental impact on educational outcomes (Condron et al. 2013), it creates concentrated poverty (Massey and Fischer 2006) and crime (Peterson and Krivo 2010). On the other hand, social integration creates greater solidarity and trust (Angell 1947), improves longevity and well-being in communities (Moen et al. 1989), benefits social mobility (Peach 2005), helps communities to be resilient to perturbations (Folke et al. 2010), and provides access to coping mechanisms (Ramirez-Valles et al. 2010) as well as lowering conflict and crime (Crutchfield et al. 1982). Social segregation disproportionately affects socially disadvantaged and underrepresented groups, whose struggles are further exacerbated by lack of urban policy coordination, disproportionate infrastructural investments (Lucas and Porter 2016, Rode et al. 2018). While the residential and neighborhood segregation remain important, urban social segregation needs to be understood and inspected in dynamics of everyday life such as social networks and mobility (Wissink et al. 2016). Studies of transportation infrastructure have revealed inequalities in mobility opportunities (Elliott and Urry 2010). Thus, many studies focused on "mobility related social exclusion" (e.g. Schwanen et al. 2015, Lucas 2012). 
To improve social resilience in urban areas it is vital to understand the level of social segregation in a city. Social segregation has two aspects: a well-studies spatial aspect known as residential segregation, and segregation on a dynamic level (Erbe 1975) that has not been extensively studied mostly due to lack of available data that captures mobility on spatial and temporal scale. The technological advancements of the last decade now present researchers with unprecedented data that can be used to innovatively study urban dynamics, one of such is social media data (e.g. Shelton et al. 2015, Gabrielli et al. 2013). The availability of data presents a vast array of opportunity for urban researchers to reveal hidden inequities of mobility patterns in urban area. Section 2.3 summarizes the relevant literature on urban mobility. Urban mobility can reveal insights into the dynamic segregation or perhaps the dynamic integration of an urban area. Dynamic segregation here goes beyond spatial segregation and refers to social segregation in everyday interactions. Dynamic integration on the other hand refers to socially diverse interactions that are not bounded by spatial constraints. This study builds a mobility network of Boston using Twitter data and investigates the racial and income segregation of the network.

\section{Urban mobility}

In recent years urban mobility studies have taken advantage of the field of network science to advance the field. Urban mobility networks are geospatial networks in which each node has a two-dimensional location property that can be used to place the node on a plane, such as a map. If the network can be placed on a map in a way that its edges do not intersect, then the network is considered planar (Barthélemy 2011). Although many spatial networks, such as an individual's travel pattern or roads networks are planar (e.g. Masucci et al. 2013), not all spatial networks depict a planar network (Barthélemy 2011). The network of this study is a non-planar network that is an aggregation of planar individuals' mobility networks.

One property of many geospatial networks is spatial embeddedness. A spatially embedded network suggests that nodes that are in spatial proximity of each other have a higher chance of being connected to each other and the connection probability of two nodes decay with increase of distance (Bullock et al. 2010). The Spatial embeddedness is mostly used in generating random graphs and the objectives are mostly mathematical to understand influence that geometric distances have on the merely topological connections (Picciolo et al. 2012). However, in real world networks, the relationship between two nodes cannot be determined merely by the spatial organization of its nodes and shared affinities and community structures can have enormous impact on the behavior of the network as well as edge distribution (Bullock et al. 2010). As the network of this study is based on actual individuals' location, this study does not look into spatially embedding the network to avoid suppressing the actual patterns that exists in the network.

Human travel patterns have been of interest to many scholars. There have been several studies on general mobility patterns confirming that human travel patterns follow a mathematically described two-parameter continuous-time random walk model, a power-law distribution (e.g., Brockmann et al. 2006; Gonzalez et al. 2008; Song et al. $2010 a, b)$. Such studies focus on travel patterns during normal circumstances when 
there are no instances of disruption. Furthermore, a few studies have focused on understanding human mobility before, during, and after natural hazards, which disrupt normal traveling patterns. For example, one study constructs London's metro infrastructure network and identifies the points of vulnerability to understand their implications on the system's resilience (Chopra et al. 2016). Wang and Taylor (2015) conducted a study that focuses on the significance of human mobility disruption during tropical typhoons to help with disaster response management. However, when considering resilience, the literature is focused on understanding the role of mobility patterns on infrastructural resilience of urban areas, ignoring the social aspect of such networks. Social resilience and diversity play a vital role in urban resilience as social exclusion is a consequence of the lack of resilience in spatial fragmentation processes (Cumming 2011). Geography is coupled with human interaction and is a key feature for contextualizing social relationships (Toole et al. 2015). Puura et al. (2018) used mobile phone call detail record (CDR) data to infer social relations as well as individual mobility and found out that the more extensive and individual's network of calling partners, the more mobile they are highlighting the role of human interaction in mobility. Lack of social resilience will affect excluded groups in many ways among which is the lack of coping mechanisms in cases of physical and socio-economic disruptions (Chaves et al. 2008).

On the other hand, the study of social networks has gained prominent attention from a different range of disciplines as well (e.g. Watts 2004; Easley and Kleinberg 2010). However, they are mainly focused on the social dimension, leaving out the interactions between humans and the physical space (Wang et al. 2011). Recently, researchers have started to treat urban mobility as urban geosocial networks and analyze their unique properties using approaches from network science. Phillips et al. (2019) used geocoded twitter data for 50 large US cities to develop a "structural connectedness" measure that is based on everyday travel patterns of people from different neighborhoods in a city and identifies whether people from different neighborhoods travel to all other neighborhoods in equal proportion. They uncover valuable information such as neighborhoods that act as hubs, lack of mobility between some neighborhoods, and the relationship between mobility and structural connectedness. In light of the new development, the aim of this study is to examine the network structure of the mobility in Boston as a case study to better understand the underlying characteristics of mobility networks on urban resilience from a social perspective.

\section{Urban mobility resilience vs. transportation system resilience}

While urban mobility and transportation systems deeply integrate and are often-times coupled with one another, they are different in essence and thus regard different resilience features. The studies on the resilience of mobility networks, especially with a focus on social aspects, requires different methodologies and tools from the ones on transportation systems. We distinguish transportation and mobility in this paper as follows: the static set of mediums by which humans move are referred to as transportation, and the movement itself is mobility. Transportation is the infrastructures that are largely in place, whereas mobility is not limited to them. 
Transportation resilience is looked upon from engineering and infrastructure perspectives and is referred to as the ability of the system to maintain its demonstrated level of service or restore itself to that level in a timely manner (Freckleton et al. 2012). Minimizing operational loss is another concern of transportation resilience (Pant 2012).

Although there have been numerous studies on resilience from different perspectives, there are few studies that focus on urban mobility resilience. Cutter et al. (2008) looks at mobility as a social system interacting with a defined geographical space and refers to resilience as the system's capacity to absorb disturbance and return to a fully functional state that advances the state of the system through learning (Adger et al. 2005; Klein et al. 2003). Fernandes (2017) refers to the resilience of urban mobility as "capacity to persist, ability to adapt, and the potential to transform the city when confronted with a threat." Wiles et al. (2012) study resilience concepts in the gerontology field and express that mobility resilience "might refer to an individual's personal mobility in terms of whether they are able to walk, whether they have a car, or whether they are physically fit, but also to broader environmental elements of mobility, such as whether there is an accessible high-quality public transport system or other forms of collective transport, whether a neighborhood has high 'walkability', or what road conditions are like and the degree to which a person feels safe to use them".

The lack of research on mobility resilience motivated this study to develop a definition that can serve as a basis of this research. For this purpose, this study looks at urban mobility in terms of one of many interconnected urban systems acknowledging that urban mobility should be looked at as a system that involves the interactions of humans with their surrounding built and ecological environment (Vermaas et al. 2011; Clegg 2000).

\section{Disruption in mobility networks vs. disruption in transportation networks}

One of the studied ways of improving the resilience state of a transportation network is through robust system architecture and introducing redundancy (Li 2016). In transportation networks, nodes are places and edges are the infrastructural means that connect the two nodes, such as railroads and streets. Having more than one path between two nodes in the network can reduce the chance of disconnection of the two nodes, although tightly coupled networks can be more vulnerable due to faster spread of failure (Vespignani 2010). Removal of a node in a transportation network -such as disruption in a train station - is another challenge that can be overcome by reaching to the destination by another mode of transportation or taking another path to a neighboring node.

Studies on mobility networks, on the contrary, usually conceptualize the nodes and edges differently from the ones on transportation networks. For example, in Phillips et al. (2019), nodes are origin and destination block groups -such as home and work locations- and edges are people who travel between two nodes regularly. Removal of a node means economic or social disruption for people who travel on the edges connected to that node. For example, natural disasters such as flooding, or man-made attacks to the area can permanently or temporarily remove a node from the network; meaning residents who depend on the removed nodes for work (or residence) will not be able to access their destination (or origin). Thus they suffer from economic and 
social consequences. This study follows a similar way to build the mobility network in Boston.

\section{Motifs in mobility networks}

Motifs are recurring patterns of connections within a network and can represent broad ranges of phenomena (Milo et al. 2002). The abundance of triadic motifs in networks is significantly correlated with stability or robustness of the network against perturbation (Scherrer et al. 2008). It is expected that human social networks have highly connected motifs to maximize information flow as opposed to, for example, a hierarchical military network that information flow occurs only in one direction (Krause et al. 2007). Schneider et al. (2013a) studied the motifs of individuals' mobility patterns in Paris using mobile phone call detail record (CDR) and found out that only 17 unique motifs explain 90\% of mobility patterns. Jiang et al. (2017) used a similar approach using CDR data to study the motifs of human mobility in Singapore and suggested using such patterns to improve urban and resource planning.

These studies have focused on individuals' mobility patterns and the resulting motifs. In this study the network is an aggregated mobility network of all the individuals in the data. Thus, the meanings behind motifs differ from the ones of individuals' movements. In a directed network there are sixteen different motifs with three nodes (they are listed in Table 3). Table 1 represents example of situations in which a certain network motif is abundant.

\section{Methodology and data}

Data

In numerous occasions, complete enumeration of data to create and analyze a realworld network is not possible. The incompleteness of data is especially true in networks that involve human mobility. However, with the popular use of smartphones and new information technologies, the availability of geocoded data is helping to resolve this problem, although not yet to the fullest. For example, a study used geocoded data from public shared photos on Flicker to study individuals' mobility patterns (Barchiesi et al. 2015). Another study used geocoded twitter data to investigate the "structural connectedness" of US cities based on everyday mobility patterns of residents (Phillips et al. 2019). The use of geocoded social media data has become very popular in urban research domain, including urban activities and environmental studies (Heikinheimo et al. 2020). A study found Twitter users to be representative of general demographics of U.S. population (Wang and Taylor 2016). Although survey-based intra-urban

Table 1 Examples of abundance of certan motif types in a mobility network

\begin{tabular}{lll}
\hline Motif & $\cdot$ &
\end{tabular}


mobility data is available for many cities, such data sources most often are not available on geographically small resolution and they lack enough detail for arriving at empirical insights (Heikinheimo et al. 2020). Twitter data has also the added potential of capturing locations other than home and work locations that are on individuals' regular travel patterns.

The use of Twitter data in understanding mobility in recent years has been prevalent, as the location-sharing technology has made it possible for researchers to take advantage of exceptional access to direct records of human activities in urban areas (Hawelka et al. 2014). Hawelka and his colleagues used almost a billion tweets to map the mobility profiles of different nations and later validated their findings using global tourism statistics and concluded that Twitter is exceptionally useful for understanding and quantifying mobility patterns (Hawelka et al. 2014). Jurdak et al. (2015) analyzed six million geo-coded tweets in Australia and demonstrated that Twitter could be a reliable source for studying human mobility patterns.

This study uses geotagged twitter data from October 2013 to March 2015. Each entry of a geotagged tweet contains the complete information of that micro-message including the user ID, tweet ID, time stamp, latitude, and longitude. The data is then aggregated to the block group defined by the U.S. Census. A user has to tweet from a specific block group 5 times or more to assume that the block group is in his/her routine travel trajectory. The final data consists of 25,585 data points. Origin and destinations have been inferred from the time of the tweets. If the user tweets any time between $8 \mathrm{pm}$ and $8 \mathrm{am}$, the location is treated as the origin. The location is treated as destination if the tweet's timestamp is within the time range of $8 \mathrm{am}$ to $8 \mathrm{pm}$. Using the timestamps to assess origin and destination is a common assumption among similar studies (e.g. Rashidi et al. 2017; Gao et al. 2014). Moreover, we used time stamps to count the number of tweets by each user from each block group that are at least $12 \mathrm{~h}$ apart from each other. For example, if a user tweets 10 times from the same block group between $6 \mathrm{pm}$ and $6 \mathrm{am}$ the next day, this is counted as one occurrence and it is most likely their home location. We look for at least $12 \mathrm{~h}$ intervals as a proxy for the timespan that most people spend at home and avoid counting home locations multiple times a day that would create self-loops. In certain hours of a day social media activity peaks (Toivonen et al. 2019) and multiple tweets by a user can produce incorrect data. In the final network of the study $62 \%$ of the users only travel between two nodes, while $31 \%$ have three nodes on their regular mobility pattern, and only $7 \%$ have four or more nodes (block groups) on their regular travel. The average separation degree for all the users is 1.38 .

A block group defined by the Census Bureau is a combination of census blocks that are collectively a subdivision of a census tract. On average there are 39 census blocks in a block group. A block is the smallest geographic entity for which tabular sample data is published by the decennial census (United States. Bureau of the Census 1994). Despite availability of data on a census block scale, this study uses block groups as nodes of the network and aggregates users' data to a block group basis to ensure ethical use of data and unfeasibility of identifying any individual based on their mobility pattern.

After cleaning the dataset, a network of mobility among the block groups is formed. In this network each node is a block group and each weighted edge represents the 
number of individuals that regularly travel between the two nodes. For this study, we excluded edges with their weights less than 10 which are the edges with fewer than 10 people traveling between a dyad of two block groups. We removed these edges so the network only focuses on more regular travel patterns. The removed edges represented $45 \%$ of the total number of edges but only $8 \%$ of the total weight of the network. In the final network there are 417 nodes and 2321 edges. There is a total of 610 block groups in Boston and the network of this study represents $68.3 \%$ of them. The distribution of the nodes covers all neighborhoods of Boston. To investigate representativeness of our data, we investigated the relationship between the population of block groups and number of users in each block group and found a linear statistically significant relationship between population and number of users with $p$-value of less than 0.05 and Rsquared of 0.69. This finding underlines that the data is representative of the size of the population. However, there could still be bias in data in terms of racial representation. Since the data covers all neighborhoods of Boston, including majority non-white neighborhoods and on the basis of previously-discussed representativeness of Twitter data, we assume that this network is representative of the population in Boston in terms of size, race, and income.

The socio-economic characteristics -such as income level and race- of each block group are attached to each node as the node attributes to understand the underlying socio-economic characteristics of the mobility network. This data is obtained from the US Census Bureau.

\section{Network measures}

The following network measures are used in this study:

- Each node is a block group. Section 3.1 contains more explanation on nodes of the network.

- Weighted edges are the number of people traveling between two nodes. $W_{i j}$ is the weight of the edge going from node $i$ to node $j$.

- Density (q) is a redundancy measure for the whole network that shows the fraction between the total number of links $(\mathrm{m})$ and the maximum possible number of links ( $\left.\frac{n \times(n-1)}{2}\right)$.

$$
q=\frac{2 m}{n \times(n-1)}
$$

- Node connectivity is the minimum number of nodes that needs to be removed for the network to be disconnected.

- Network motifs are recurring patterns of connections in a network.

- Network communities are locally dense subgraphs of the network that are formed in real-world networks (Yang and Leskovec 2015).

\section{Network communities}

The communities of this network are detected using Louvain modularity algorithm. Each community is a combination of nodes which are more likely to be connected to each other rather than to nodes from outside of their community. In the context of a mobility network, people who live in the same network community are more likely to 
mobilize within their own network community. We would like to emphasize that a network community is merely characterized by modularity which uses edge weights and distribution of the weights among different nodes as features. The network community concept is not influenced by geographical neighborhoods or and should not be confused with social and geographical communities.

Modularity in a network is a measure to assess the network community structure. Modularity is based on the edge connectedness in the communities where the nodes in the same community are more likely to connect to each other rather than to nodes from different communities. Thus, a community is a locally relatively dense subgraph of the network. The optimal community structure happens when the modularity of the network is maximized. Modularity for the whole network can be calculated through the following equation (Barabási 2016):

$$
M=\sum_{c=1}^{n_{c}}\left[\frac{L_{c}}{L}-\left(\frac{k_{c}}{2 L}\right)^{2}\right]
$$

Where $L$ is the total weights of all the links in the network, $L_{c}$ is the total weights of all the links within the community $C$ and $k_{c}$ is the total degree in that community. The community structure in this study is detected using the Louvain algorithm (Blondel et al. 2008). This algorithm, starts with each node as a separate community and calculated modularity for the while network. At each iteration the algorithm merges two communities and recalculates modularity. If the modularity had increased, the algorithm keeps the nodes in the same community and repeats this process. If the modularity had decreased, the algorithm removes the node from the last step from the community. The model iterates over these steps many times until it arrives at the highest modularity score, at which point moving one node from a community to another will only decrease modularity. The complexity of this model and the number of iterations increases as $n \log ^{2} n$ with increase in $n$ as number of nodes (Lancichinetti and Fortunato 2009).

\section{Motif detection}

The motifs of this network have been identified using Cytoscape, and opensource platform for complex network analysis (Shannon et al. 2003). To understand the level of abundance of a certain motif, z-scores are used. Z-scores are defined as the difference in the frequency of the motif and its mean frequency in a set of sufficiently large randomized networks divided by the standard deviation of frequency values in the randomized network (Milo et al. 2002). In simpler words, the number of appearances of a certain motif in a network is compared to the number of its appearances in a random network of the same size. In this study each motif type in a community is compared to a set of 10,000 randomized networks with degree preservation to arrive at the Z-scores. $Z$-scores are then normalized to enable comparison of their significance across different communities with different sizes and are defined as (Ma et al. 2008):

$$
N_{i}=\frac{Z_{i}}{\sum \sqrt{Z_{i}^{2}}}
$$


The normalized z-scores of each motif along with more discussions are presented in section 4.4.

\section{Results}

\section{Mobility communities}

The outcome of Louvain modularity algorithm divides the nodes into 21 different communities, with some of the communities consisting of less than 5 nodes. This study only focuses on the 7 major detected communities. A network community represents a subset of nodes that are densely connected to each other and a node is more probable to be connected to another node in the same community compared to nodes outside of the community. In the context of this mobility network, traveling within each community is more probable than traveling outside of the community. Each node (block group) in the network has sociodemographic attributes such as percentage of white population, percentage of non-white population, median income, and educational attainments. These attributes are used to uncover social unbalances in this network. Figure 1a highlights the 7 biggest network communities in different colors. Mobility is more probable between nodes that are in the same community. G0 in orange includes 64 nodes, G1 in pink includes 60 nodes, G2 includes 57 nodes in green, G3 includes 54 nodes in blue, G4 includes 51 nodes in purple, and G5 and G6 include 50 yellow and 30 red nodes respectively. Although the network communities only follow network structural measures such as modularity, the nodes in each community are also geographically clustered. Figure $1 \mathrm{~b}$ shows nodes that more than $50 \%$ of their population are either white or other races. Figure $1 \mathrm{a}$ and $\mathrm{b}$ share some resemblance in the segregation patterns. The percentage of white population versus other races in each community is shown in Fig. 1c. Figure 2 shows the distribution of white and non-white population of Boston among different communities with the number of people on the left vertical axis and the percentage on the right axis. Other than G3, the six other mobility communities are not racially balanced, indicating that it is more probable that minorities and whites travel within themselves. This social mobility segregation is not present in G3 where the whites and other races are more in balance with one another.

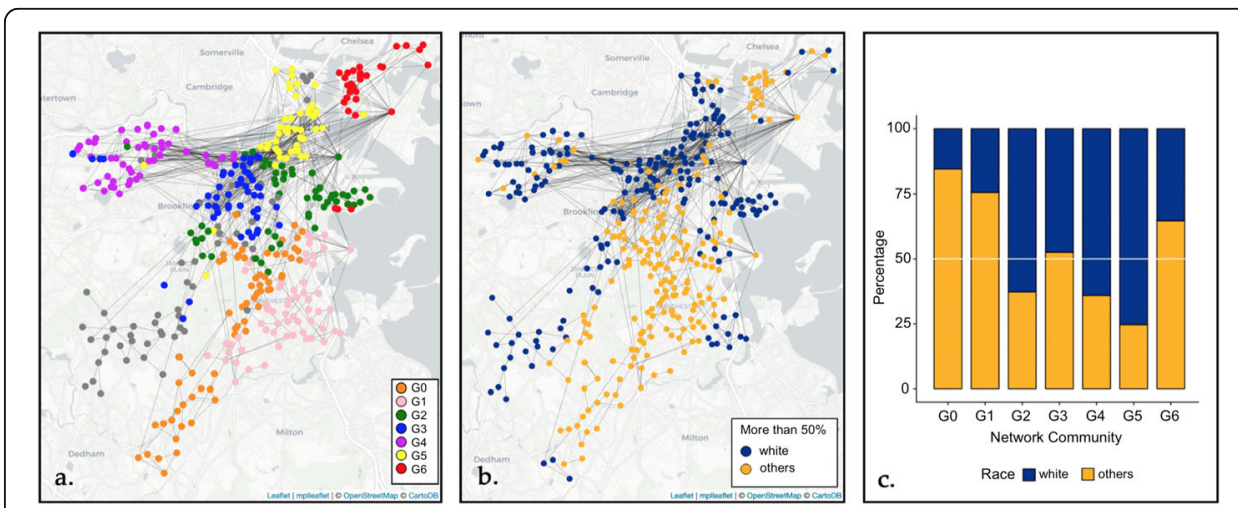

Fig. 1 a shows the community structure of the network based on modularity, detected via the Louvain algorithm. b shows the nodes with more than $50 \%$ white vs. nodes with more than $50 \%$ minorities. c shows the percentage of white vs. other races in each community 


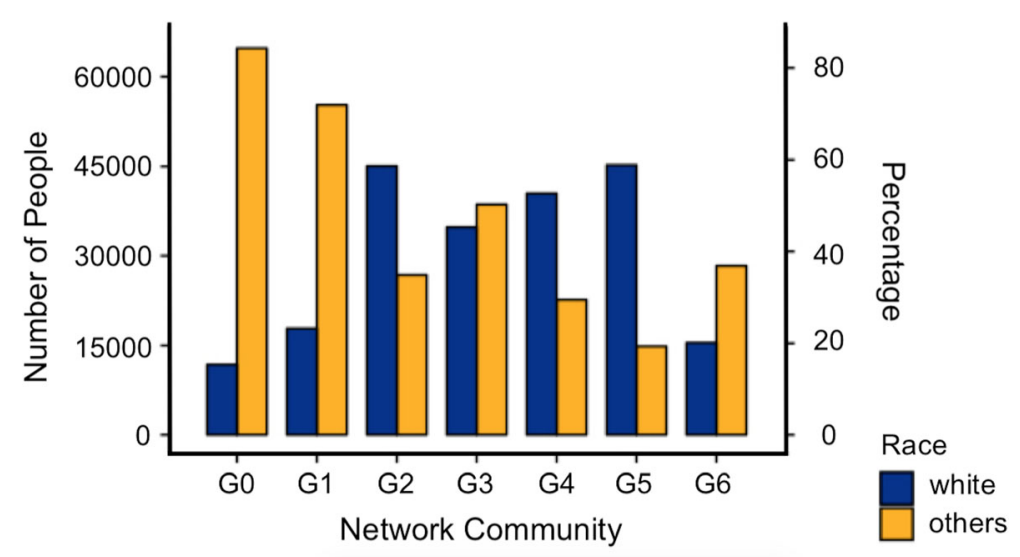

Fig. 2 The distribution of white and non-white population of Boston among different communities

There are three points that are worth mentioning here:

1. The mobility communities are spatially governed. Even with the current influence of technology on our everyday lives, geography is still very much valid. Although information technology shrinks time and space in many ways, the geographic space still plays a significant role in social phenomena (Sheldon 2014). The findings align with the ones from previous research. For example, Wang et al. (2018) found that although marginalized residents travel longer distances on a daily basis, they are also less likely to travel to non-poor white neighborhoods. Phillips et al. (2019) have also shown that spatial social segregation affects the connectedness of the neighborhoods and limits travels between two neighborhoods with different socioeconomic status.

2. We also observe that the composition of G3 community suggests a high level of social cohesion -social cohesion is the extent of connectedness among groups in society (Kawachi and Berkman 2000). We hypothesize that the spatial placement of this network community and its nodes (block groups) to numerous diverse services play a key role in this phenomenon. For example, the nodes in G3 are clustered around boundaries of neighborhoods such as Back Bay (a hub for financial and leisure activities in Boston), Longwood (a hub for medical services and sciences), Northeastern University, and Boston University. The diversity of people who travel to these educational, medical, and leisure hubs create a diverse mobility community. The drivers of such social cohesion observed in the mobility network deserve further investigation.

3. Communities such as G0, G1, and G5 are more segregated than other communities. G0 and G1 are majority poor black suburban neighborhoods of Boston, including Dorchester, Mattapan, Roxbury, and Dudley Square neighborhoods, which all have locally low median housing values and median household income (Compared to other neighborhoods of Boston). G5 includes non-poor white areas of the city, including downtown, financial district, Charlestown, and Beacon Hill areas. These neighborhoods have some of the highest median household incomes and median housing values in the city of Boston. There can be numerous factors in play other than income and housing values, such as 
accessibility to public transportation, or availability of job opportunities, which will be the focus of future work.

\section{Other characteristics of the network communities}

Sociodemographic attributes of each node (block group) other than race, which was discussed in section 4.1 is used to uncover inequities of this network. These features along with some other main network properties of the communities are presented in Table 2. Community G3 which is racially more balanced than other communities (ratio of white to non-white population is close to 1), has the highest density, indicating that the subgraph is more well-connected within itself compared to others, a fact that is also reflected in its highest average degree. It has the highest node connectivity among the seven communities, meaning that 6 nodes need to be removed to fragment the subgraph into two parts. Again, majorly white communities have more network structural advantages, such as higher density and node connectivity, and support community resilience to a higher degree than communities with more minority population. For example, G0 is a majority white community with a white to non-white ration of 3 . It has considerably higher median income and higher percentage of college-educated population. It has the second highest average node degree and node connectivity which allows the community to be more resilient to perturbations. On the other hand, communities such as G0 or G1 which high percentage of non-white population and low median income, have considerably lower network densities and average node degrees, both indicators of not well-connected communities. Their node connectivity is 1 , meaning by just removing 1 node the community can become disconnected. Such communities are vulnerable to perturbations.

\section{Multinomial regression}

We performed multinomial regression analysis in which the network communities are captured in a multinomial dependent variable with 7 categories. The independent variables are socioeconomic characteristics of the nodes including, percentage of white population, percentage of black population, percentage of other races, and median household income. Multinomial regression, a type of logit regression is used to find causality between a categorical independent variable with two or more categories, and a group of dependent variables.

Table 2 Other characteristics of the network communities

\begin{tabular}{llllllll}
\hline & G0 & G1 & G2 & G3 & G4 & G5 & G6 \\
\hline Network Measures & & & & & & & \\
$\quad$ Density & 0.014 & 0.022 & 0.028 & 0.078 & 0.056 & 0.064 & 0.067 \\
$\quad$ Node connectivity & 1 & 1 & 3 & 6 & 4 & 2 & 2 \\
$\quad$ Average node degree & 2.193 & 2.879 & 4.8 & 10.642 & 7.098 & 9.282 & 4.967 \\
$\quad$ Highest node degree & 7 & 13 & 55 & 43 & 28 & 47 & 37 \\
Socio-economic characteristics & & & & & & & \\
$\quad$ Median household income (USD) & 20,126 & 22,286 & 52,645 & 20,629 & 29,274 & 71,017 & 25,642 \\
$\quad$ Percentage college educated & 18.11 & 24.99 & 50.06 & 44.81 & 59.80 & 63.00 & 24.67 \\
$\quad$ White to non-white ratio & 0.219 & 0.351 & 1.857 & 0.923 & 2.125 & 3 & 0.562 \\
\hline
\end{tabular}


There are 366 nodes in the network communities. We randomly chose 183 nodes which will account for $50 \%$ of the network communities as the input to train our model. Then we used the other half of the network as a test dataset. Because the number of observations in our dataset is small (366 data points in total) we chose a 50-50 train and test split in order to have a big-enough test set to allow for meaningful accuracy discussion. The regression model achieved $63.4 \%$ accuracy. Figure 3 is the confusion matrix of the model that shows the number of nodes that have been placed correctly on the right communities using the regression model on the diagonal cells. This result indicates that more than $60 \%$ of the dynamic segregation that is present in Boston's mobility network can be explained by the spatial racial and income segregation of the city.

\section{Network motifs}

Table 3 presents the number of different triadic motifs in the identified network communities. Communities G0 through G6 have 64, 58, 58, 59, 60, 59, and 42 nodes respectively. Because of the closeness of the community sizes it is therefore possible to compare the number of motif occurrences in the communities, which are presented in Table 3. However, Z-score for each motif in different networks was obtained as a measure of statistical significance, which is the basis for the heatmap in Fig. 3.

Figure 4 represents a heatmap of the significance of different motif type in each community. The Z-score and normalized Z-scores of the motifs represented in the heatmap are all negative, indicating that all motifs have low presence in the network as compared to randomized networks albeit with significant differences. The low presence could be a result of lack of connectivity or presence of hubs. As shown in Fig. 3, occurrences of all motif types especially motifs with more edges- are more significant in

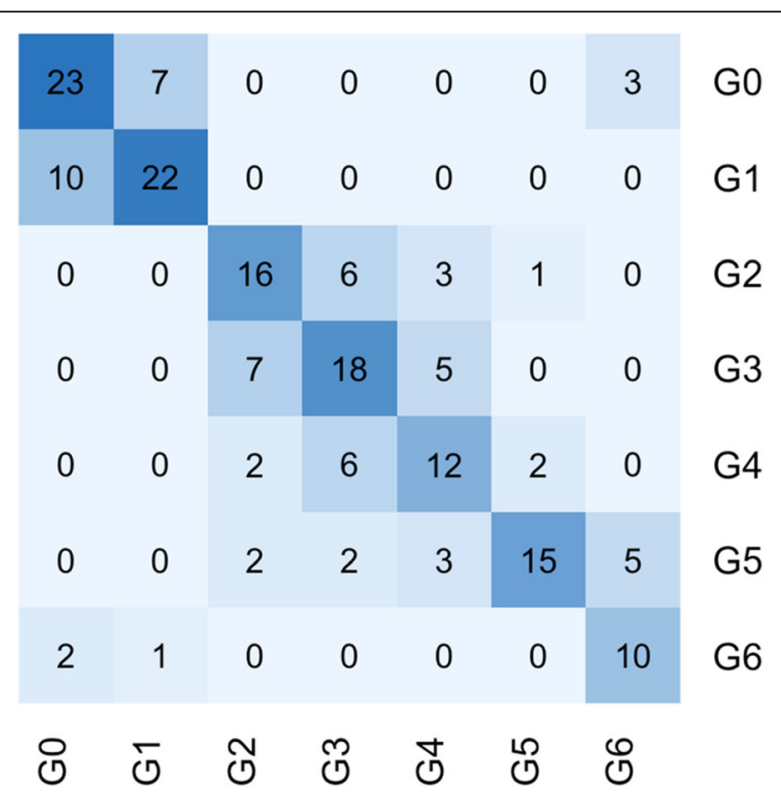

Fig. 3 Confusion matrix resulting from multinomial regression analysis in which the dependent variable is network communities and the independent variables are percentage of white population, percentage of black population, percentage of other races, and median household income 
Table 3 Number of appearances of each triadic motif in identified network communities

\begin{tabular}{|c|c|c|c|c|c|c|c|c|}
\hline Code & Motif & G0 & G1 & G2 & G3 & G4 & G5 & G6 \\
\hline 003 & $\therefore$ & 38,439 & 30,686 & 25,273 & 17,434 & 15,980 & 14,223 & 2728 \\
\hline 012 & $\therefore$. & 3047 & 2883 & 3373 & 4494 & 3150 & 3663 & 1082 \\
\hline 102 & I. & 118 & 539 & 329 & 1874 & 1153 & 1009 & 55 \\
\hline 021D & $\therefore$ & 16 & 25 & 31 & 99 & 47 & 77 & 42 \\
\hline $021 \mathrm{U}$ & $\therefore$ & 17 & 23 & 143 & 127 & 113 & 166 & 72 \\
\hline $021 C$ & $\therefore$ & 20 & 20 & 46 & 145 & 78 & 109 & 42 \\
\hline $111 \mathrm{D}$ & $\triangle$ & 3 & 16 & 32 & 212 & 132 & 125 & 19 \\
\hline $111 \mathrm{U}$ & $\Lambda$ & 3 & 19 & 15 & 157 & 45 & 94 & 6 \\
\hline О30 T & $\triangle$ & 0 & 4 & 10 & 48 & 14 & 27 & 10 \\
\hline O30C & $\Delta$ & 1 & 0 & 0 & 1 & 1 & 1 & 1 \\
\hline 201 & $\Lambda$ & 0 & 1 & 0 & 54 & 26 & 24 & 1 \\
\hline $120 D$ & $\triangle$ & 0 & 1 & 4 & 42 & 19 & 25 & 2 \\
\hline $120 \mathrm{U}$ & $\Delta$ & 0 & 1 & 2 & 33 & 21 & 16 & 0 \\
\hline $120 C$ & $\triangle$ & 0 & 2 & 1 & 18 & 8 & 13 & 0 \\
\hline 210 & $\triangle$ & 0 & 0 & 1 & 42 & 25 & 22 & 0 \\
\hline 300 & $\Delta$ & 0 & 0 & 0 & 24 & 13 & 6 & 0 \\
\hline
\end{tabular}

communities G3, G4, and G5, while communities G1, G2, and G6 mostly have less firmly connected triads, and community G0 has the least number of every triadic motif type. However, Table 3 shows that the number of unconnected triple nodes -motif code 003- in communities G0 and G1 is significantly higher than other communities. The nodes in unconnected triads are not isolated nodes and they are connected to other nodes in (and in some cases out of) their community. However, the significant presence of unconnected triads shows the community is not very well connected and thus vulnerable to perturbations.

Moreover, communities with the least dense triads G0, G1, and G6 are the communities with a higher percentage of the non-white population. Mobility communities with a higher percentage of white population -such as G4-and more racially balanced communities - such as G3- tend to have a more significant presence of highly connected triadic motifs. Densely connected triadic motifs facilitate community and social

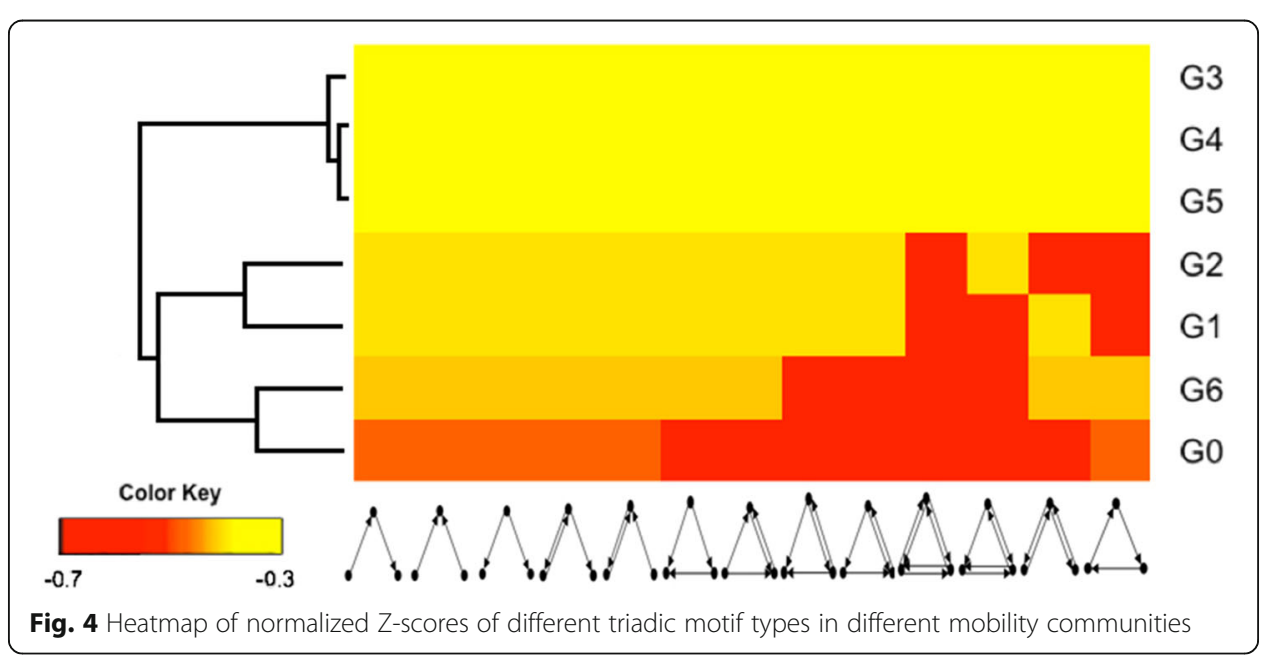


resilience through knowledge sharing and interactions beyond cultural and economic boundaries.

\section{Bigger motifs}

The highest frequency motifs of degree 4, 5 and 6 in the entire network and seven major communities are shown in Table 4. Each motif's frequency is listed beneath it. There are a few major observations here worth mentioning. First, all motifs are low in density indicating that major parts of the network have low densities despite the presence of some locally dense subgraphs.

Secondly, the frequent motifs in G2 and G3, and to a lesser degree G4 are very similar to the frequent motifs of the entire network, showing the domination of these three communities in shaping the patterns of connection in the entire network.

Thirdly, the patterns of the frequent motifs in G0 and G1 significantly differ from other communities. In G0 and G1 the flows tend to disperse from one node to others whereas in other communities and more specifically G2, G3, and G5 flows merge and meet in one node. This fact is an indication of fewer local attractions in G0 and G1 such as financial districts that can supply the job demands of the community. G2, G3 and G5, which have more attractive central nodes, are located in areas that are medical or education or financial hubs in Boston area.

G4 is the only community with reciprocated flows, also known as bi-flows (two-directional flows) in the most frequent motifs. Bi-flows are strong features in a mobility community as they show the co-independence of neighborhoods on one another and can improve socio-economic resilience of the community. G4 is the Allston neighborhood of Boston, which includes parts of Boston University as an educational hub, many restaurants and bars as leisure hubs, and is home to socio-demographically diverse residents.

Finally, G6 is the East Boston neighborhood, which includes Boston Logan International Airport. The majority Hispanic population of East Boston has created a very locally dense subgraph, which has a higher density than any other non-white community in the network.

\section{Limitations and future research}

Although using geocoded social media data in research is becoming increasingly popular (e.g. Matamalas et al. 2016; Achrekar et al. 2011; Tremayne 2014; Grandjean 2016), the use of twitter data encompasses limitations. While researchers have explored the representativeness of Twitter data (Mislove et al. 2011; Wang and Taylor 2015), the

Table 4 Most frequent motifs of sizes 4, 5 and 6 in the network and its communities

\begin{tabular}{|c|c|c|c|c|c|c|c|c|}
\hline & Entire Network & G0 & G1 & G2 & G3 & G4 & G5 & $\mathrm{G} 6$ \\
\hline & 1. & $\therefore$ & $\therefore$ &. & 1. & . & 1. & 1 \\
\hline \multirow[t]{2}{*}{ Frequency } & 1354 & 205 & 41 & 352 & 355 & 215 & 327 & 202 \\
\hline & $\because$ & $\therefore$ & $B$ & 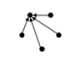 & $\theta$ & $\therefore$ & $\therefore$ & $\therefore$ \\
\hline \multirow[t]{2}{*}{ Frequency } & 1894 & 549 & 27 & 692 & 668 & 409 & 669 & 329 \\
\hline & $\therefore$ & $\therefore$ & 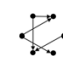 & 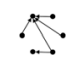 & 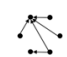 & $\therefore$ & $\therefore$ & $\leadsto$ \\
\hline Frequency & 3012 & 1216 & 29 & 1684 & 1128 & 484 & 1114 & 381 \\
\hline
\end{tabular}


geotagged Twitter data might not be representative of the whole demographics. However, this study serves as a mandatory basis of advancing the field. Future research should compare results from this study to the ones derived from other data sets. Also, although we limit our analysis to frequent visits, there could be bias in the visits reported in geotagged Twitter data which could impact the structure of the mobility networks in Boston. Moreover, our analysis on urban resilience has been focusing on mobility network while other aspects of the system introduced in section 1.2. deserve future investigations. Moreover, in the study of motifs, the presence or absence of highly connected motifs can be an indicator of imbalance in resource distribution such as job opportunities. Our future efforts will focus on investigating the underlying shaping factors of such imbalance.

\section{Conclusion}

Studying the resilience of urban areas is not a trivial task but can be achieved through studying urban systems. This study focuses on social resilience aspect of Boston's urban mobility network. This study builds the aggregated mobility network of Boston as one of the urban systems that lie within the intersection of many disciplines. The essence of such a network is to investigate the interactions between different parts of the city with a block-group resolution. The resulting network illustrates that the spatial social segregation in Boston is also present in the network community structures where six out of seven main mobility communities in the network are racially and economically segregated, indicating that racial minorities and lower-income demographics are more likely to move within themselves rather than interacting beyond the economic and racial boundaries. The results of this study align with findings of Dannemann et al. (2018) where they used cell phone data to uncover dynamic social segregation in Santiago, Chile. The result of the multinomial regression model indicates that spatial racial and income segregation in Boston can be used to explain 63\% of the segregation in mobility network communities. Moreover, the study of motifs of the network communities shows dissimilar patterns of interaction in different communities. The analysis of triadic motifs shows that minority dominated communities lack well-connected triads. Thus, the interactions between nodes in those communities are weaker than the white dominated communities in which the abundance of denser triads are more significant. Moreover, in minority dominated network communities the study of higher degree motifs reveals common dispersal patterns where people regularly tend to scatter to different parts. However, in majorly white communities there are more nodes that attract flows. In those communities, people tend to gather in a central node. This finding can potentially be an indicator of the difference in economic opportunities available to different communities. The only racially balanced network community (G3) has the most significant presence of dense triadic motifs and reflects strong interaction between nodes that can possibly help improve community, social, and economic resilience. Overall, the findings of this study stress the imbalance in social resilience that is happening through mobility. Dynamic racial segregation, such as the ones shown in this study, can limit the ability of people interacting beyond social and economic boundaries and potentially prohibits upward mobility for minorities. 


\section{Acknowledgements}

Not Applicable.

\section{Authors' contributions}

S.M. designed computational framework, analyzed the data, and took the lead on writing the manuscript. Q.W. acquired the data, interpreted the findings and provided feedback on the manuscript. Both authors contributed shaping the research and analysis and developing the manuscript. Both authors approve the submitted manuscript.

\section{Funding}

This work was supported by National Science Foundation Grant HDBE-1761950; Northeastern Tier 1 Project on "Neighborhood Connectivity and Social Inequality"; and Global Resilience Institute Project on "Geosocial Network Resilience".

\section{Availability of data and materials}

The data that support the findings of this study are openly available at https://github.com/SaharMirzaee/project1/ $\mathrm{blob} / \mathrm{master} / \mathrm{bostondata.csv}$

\section{Competing interests}

The authors declare that they have no competing interests.

\section{Author details}

${ }^{1}$ Department of Public Policy and Urban Affairs, Northeastern University, Boston, MA 02115, USA. ${ }^{2}$ Department of Civil and Environmental Engineering, Northeastern University, Boston, MA 02115, USA.

\section{Received: 8 April 2020 Accepted: 6 September 2020}

Published online: 09 October 2020

\section{References}

Achrekar H, Gandhe A, Lazarus R, Yu SH, Liu B (2011) Predicting flu trends using twitter data. In 2011 IEEE conference oncomputer communications workshops (INFOCOM WKSHPS). IEEE, Shanghai, pp. 702-707

Adey P (2010) Aerial life: spaces, mobilities, affects. Wiley-Blackwell, West Sussex

Adger WN, Hughes TP, Folke C, Carpenter SR, Rockström J (2005) Social-ecological resilience to coastal disasters. Science 309(5737):1036-1039

Amir S, Kant V (2018) Sociotechnical resilience: a preliminary concept. Risk Anal 38(1):8-16

Angell RC (1947) The social integration of American cities of more than 100,000 population. Am Sociol Rev 12(3):335-342

Bagrow JP, Wang D, Barabasi A-L (2011) Collective response of human populations to large-scale emergencies. PLoS One 6: e17680

Barabási A-L (2016) Network science. Cambridge university press, Cambridge

Barchiesi D, Preis T, Bishop S, Moat HS (2015) Modelling human mobility patterns using photographic data shared online. R Soc Open Sci 2(8):150046

Barthélemy M (2011) Spatial networks. Phys Rep 499(1-3):1-101

Blondel VD, Guillaume JL, Lambiotte R, Lefebvre E (2008) Fast unfolding of communities in large networks. J Stat Mech Theory Exp 2008(10):P10008

Brockmann D, Hufnagel L, Geisel T (2006) The scaling laws of human travel. Nature 439(7075):462

Bullock S, Barnett L, Di Paolo EA (2010) Spatial embedding and the structure of complex networks. Complexity 16(2):20-28

Candia J, González MC, Wang P, Schoenharl T, Madey G, Barabási A-L (2008) Uncovering individual and collective human dynamics from mobile phone records. J Phys A Math Theor 41:224015

Chaves LF, Cohen JM, Pascual M, Wilson ML (2008) Social exclusion modifies climate and deforestation impacts on a vectorborne disease. PLoS Negl Trop Dis 2(2):e176

Cho E, Myers SA, Leskovec J (2011) Friendship and mobility: user movement in location-based social networks. in 1082-1090. ACM. https://doi.org/10.1145/2020408.2020579

Chopra SS, Dillon T, Bilec MM, Khanna V (2016) A network-based framework for assessing infrastructure resilience: a case study of the London metro system. J R Soc Interface 13(118):20160113

Clegg CW (2000) Sociotechnical principles for system design. Appl Ergon 31(5):463-477

Coaffee J (2008) Risk, resilience, and environmentally sustainable cities. Energy Policy 36(12):4633-4638

Condron DJ, Tope D, Steidl CR, Freeman KJ (2013) Racial segregation and the black/white achievement gap, 1992 to 2009. Sociol Q 54(1):130-157

Crutchfield RD, Geerken MR, Gove WR (1982) Crime rate and social integration the impact of metropolitan mobility. Criminology 20(3-4):467-478

Cumming GS (2011) Spatial resilience: integrating landscape ecology, resilience, and sustainability. Landsc Ecol 26(7):899-909

Cutter SL, Barnes L, Berry M, Burton C, Evans E, Tate E, Webb J (2008) A place-based model for understanding community resilience to natural disasters. Glob Environ Chang 18(4):598-606

Cuttone A, Lehmann S, González MC (2018) Understanding predictability and exploration in human mobility. EPJ Data Science 7:1-17

Dannemann T, Sotomayor-Gómez B, Samaniego H (2018) The time geography of segregation during working hours. R Soc Open Sci 5(10):180749

Easley D, Kleinberg J (2010) Networks, crowds, and markets, vol 8. Cambridge university press, Cambridge

Elliott A, Urry J (2010) Mobile lives. Routledge.

Erbe BM (1975) Race and socioeconomic segregation. Am Sociol Rev 40(6):801-812

Fernandes VA, Rothfuss R, Hochschild V, Silva MAVD, Silva WRD, Steiniger S, Santos TFD (2019) Urban resilience in the face of fossil fuel dependency: the case of Rio de Janeiro's urban mobility. urbe. Revista Brasileira de Gestão Urbana pp. 11. 
Fernandes VA (2017) Resilience of urban mobility in the face of fossil fuel dependency: an empirical study of Rio de Janeiro. Eberhard Karls Universität Tübingen

Folke C, Carpenter SR, Walker B, Scheffer M, Chapin T, Rockström J (2010) Resilience thinking: integrating resilience, adaptability and transformability. Ecol Soc 15(4):20

Fonseca X, Lukosch S, Brazier F (2019) Social cohesion revisited: a new definition and how to characterize it. Innov Eur J Soc Sci Res 32(2):231-253

Freckleton D, Heaslip K, Louisell W, Collura J (2012) Evaluation of resiliency of transportation networks after disasters. Transportation Res Rec J Transportation Res Board 2284:109-116

Gabrielli S, Maimone R, Forbes P, Masthoff J, Wells S, Primerano L et al (2013) Designing motivational features for sustainable urban mobility. In: CHI'13 Extended Abstracts on Human Factors in Computing Systems, pp 1461-1466

Gao S, Yang JA, Yan B, Hu Y, Janowicz K, McKenzie G (2014) Detecting origin-destination mobility flows from geotagged tweets in greater Los Angeles area. In: Proceedings of the eighth international conference on geographic information science, pp 1-4

Gonzalez MC, Hidalgo CA, Barabasi A-L (2008) Understanding individual human mobility patterns. Nature 453(7196):779

Grandjean M (2016) A social network analysis of twitter: mapping the digital humanities community. Cogent Arts Humanit $3(1): 1171458$

Grimm NB, Faeth SH, Golubiewski NE, Redman CL, Wu J, Bai X, Briggs JM (2008) Global change and the ecology of cities. Science 319(5864):756-760

Hawelka B, Sitko I, Beinat E, Sobolevsky S, Kazakopoulos P, Ratti C (2014) Geo-located twitter as proxy for global mobility patterns. Cartogr Geogr Inf Sci 41(3):260-271

Heikinheimo V, Tenkanen H, Bergroth C, Järv O, Hiippala T, Toivonen T (2020) Understanding the use of urban green spaces from user-generated geographic information. Landsc Urban Plan 201:103845

Hill E, Clair TS, Wial H, Wolman H, Atkins P, Blumenthal P et al (2012) Economic shocks and regional economic resilience. In: Urban and regional policy and its effects: Building resilient regions. Brookings Institution Press, Washington, pp 193-274

Jiang S, Ferreira J, Gonzalez MC (2017) Activity-based human mobility patterns inferred from mobile phone data: a case study of Singapore. IEEE Trans Big Data 3(2):208-219

Jurdak R, Zhao K, Liu J, AbouJaoude M, Cameron M, Newth D (2015) Understanding human mobility from twitter. PLoS One 10(7):e0131469

Kawachi I, Berkman L (2000) Social cohesion, social capital, and health. Soc Epidemiol 174(7)

Klein RJ, Nicholls RJ, Thomalla F (2003) Resilience to natural hazards: how useful is this concept? Glob Environ Change Part B Environ Hazards 5(1):35-45

Krause J, Croft DP, James R (2007) Social network theory in the behavioural sciences: potential applications. Behav Ecol Sociobiol 62(1):15-27

Lancichinetti A, Fortunato S (2009) Community detection algorithms: a comparative analysis. Phys Rev E 80(5):056117

Li D (2016) Resilience of spatial networks. In: Complex Systems and Networks. Springer, Berlin, Heidelberg, pp 79-106

Liang X, Zheng X, Lv W, Zhu T, Xu K (2012) The scaling of human mobility by taxis is exponential. Physica A Stat Mech Appl 391:2135-2144

Lucas K (2012) Transport and social exclusion: Where are we now? Transp Policy 20:105-113

Lucas K, Porter G (2016) Mobilities and livelihoods in urban development contexts: Introduction. J Transp Geogr 55:129-131

Ma Y, He K, Liu J (2008) Network motifs in object-oriented software systems. Discrete and Impulsive Systems (Series B: Applications and Algorithms) 14(S6):166-172

Massey DS, Fischer MJ (2006) The effect of childhood segregation on minority academic performance at selective colleges. Ethn Racial Stud 29(1):1-26

Masucci AP, Stanilov K, Batty M (2013) Limited urban growth: London's street network dynamics since the 18th century. PLoS One 8(8):e69469

Matamalas JT, De Domenico M, Arenas A (2016) Assessing reliable human mobility patterns from higher order memory in mobile communications. J R Soc Interface 13(121):20160203

Milo R, Shen-Orr S, Itzkovitz S, Kashtan N, Chklovskii D, Alon U (2002) Network motifs: simple building blocks of complex networks. Science 298(5594):824-827

Mislove A, Lehmann S, Ahn YY, Onnela JP, Rosenquist JN (2011) Understanding the demographics of twitter users. ICWSM $11(5$ th):25

Moen P, Dempster-McClain D, Williams RM Jr (1989) Social integration and longevity: an event history analysis of women's roles and resilience. Am Sociol Rev 54(4):635-647

Noulas A, Scellato S, Lambiotte R, Pontil M, Mascolo CA (2012) Tale of many cities: universal patterns in human urban mobility. PLoS One 7:e37027

Pant SB (2012) Transportation network resiliency: a study of self-annealing

Paton D, Johnston D (2001) Disasters and communities: vulnerability, resilience and preparedness. Disaster prevention and management. Int J 10(4):270-277

Peach C (2005) Social integration and social mobility: spatial segregation and intermarriage of the Caribbean population in Britain. In: Ethnicity, social mobility and public policy: comparing the US and UK, pp 178-203

Peterson RD, Krivo L (2010) Divergent social worlds: neighborhood crime and the racial-spatial divide. Russell Sage Foundation, New York

Phillips NE, Levy BL, Sampson RJ, Small ML, Wang RQ (2019) The social integration of American cities: network measures of connectedness based on everyday mobility across neighborhoods. Sociol Methods Res. Retrived from: https://doi.org/10. $1177 / 0049124119852386$.

Picciolo F, Squartini T, Ruzzenenti F, Basosi R, Garlaschelli D (2012) The role of distances in the World Trade Web. In: 2012 Eighth international conference on signal image technology and internet based systems. IEEE, Sorrento-Naples, pp 784792

Puura A, Silm S, Ahas R (2018) The relationship between social networks and spatial mobility: a Mobile-phone-based study in Estonia. J Urban Technol 25(2):7-25 
Ramirez-Valles J, Kuhns LM, Campbell RT, Diaz RM (2010) Social integration and health: community involvement, stigmatized identities, and sexual risk in Latino sexual minorities. J Health Soc Behav 51(1):30-47

Rashidi TH, Abbasi A, Maghrebi M, Hasan S, Waller TS (2017) Exploring the capacity of social media data for modelling travel behaviour: opportunities and challenges. Transportation Res Part C Emerg Technol 75:197-211

Rode P, da Cruz NF (2018) Governing urban accessibility: moving beyond transport and mobility. Appl Mobilities 3(1):8-33

Scherrer A, Borgnat P, Fleury E, Guillaume JL, Robardet C (2008) Description and simulation of dynamic mobility networks. Comput Netw 52(15):2842-2858

Schneider CM, Belik V, Couronné T, Smoreda Z, González MC (2013a) Unravelling daily human mobility motifs. J R Soc Interface 10(84):20130246

Schneider CM, Rudloff C, Bauer D, González MC (2013b) Daily travel behavior: Lessons from a week-long survey for the extraction of human mobility motifs related information

Schwanen T, Lucas K, Akyelken N, Solsona DC, Carrasco JA, Neutens T (2015) Rethinking the links between social exclusion and transport disadvantage through the lens of social capital. Transp Res A: Policy Pract 74:123-135

Shannon P, Markiel A, Ozier O, Baliga NS, Wang JT, Ramage D et al (2003) Cytoscape: a software environment for integrated models of biomolecular interaction networks. Genome Res 13(11):2498-2504

Sheldon JB (2014) Geopolitics and cyber power: why geography still matters. Am Foreign Policy Interests 36(5):286-293

Shelton T, Poorthuis A, Zook M (2015) Social media and the city: Rethinking urban socio-spatial inequality using usergenerated geographic information. Landscape Urban Plann 142:198-211

Song C, Koren T, Wang P, Barabási A-L (2010a) Modelling the scaling properties of human mobility. Nat Phys 6:818

Song C, Qu Z, Blumm N, Barabási A-L (2010b) Limits of predictability in human mobility. Science 327(5968):1018-102

Toivonen, T., Heikinheimo, V., Fink, C., Hausmann, A., Hiippala, T., Järv, O., ... \& Di Minin, E. (2019). Social media data for conservation science: a methodological overview. Biol Conserv, 233, 298-315

Toole JL, Herrera-Yaqüe C, Schneider CM, González MC (2015) Coupling human mobility and social ties. J R Soc Interface 12(105):20141128

Tremayne M (2014) Anatomy of protest in the digital era: a network analysis of twitter and Occupy Wall street. Soc Mov Stud 13(1):110-126

United States (2017) The National Security Strategy of the United States of America. President of the U.S, Washington

United States. Bureau of the Census. (1994) Geographic areas reference manual. US Dept. of commerce, economics and statistics administration, Bureau of the Census

Vermaas P, Kroes P, van de Poel I, Franssen M, Houkes W (2011) A philosophy of technology: from technical artefacts to sociotechnical systems. Synth Lect Eng Technol Soc 6(1):1-134

Vespignani A (2010) Complex networks: the fragility of interdependency. Nature 464(7291):984

Vespignani A (2012) Modelling dynamical processes in complex socio-technical systems. Nat Phys 8(1):32

Wang D, Pedreschi D, Song C, Giannotti F, Barabasi AL (2011) Human mobility, social ties, and link prediction. In: Proceedings of the 17th ACM SIGKDD international conference on Knowledge discovery and data mining. Acm, San Diego, pp 11001108

Wang Q, Phillips NE, Small ML, Sampson RJ (2018) Urban mobility and neighborhood isolation in America's 50 largest cities. Proc Natl Acad Sci 115(30):7735-7740

Wang Q, Taylor JE (2015) Resilience of human mobility under the influence of typhoons. Procedia Eng 118:942-949

Wang Q, Taylor JE (2016) Process map for urban-human mobility and civil infrastructure data collection using geosocial networking platforms. J Comput Civ Eng 30(2):04015004

Watts DJ (2004) Six degrees: the science of a connected age. WW Norton \& Company, New York

Wiles JL, Wild K, Kerse N, Allen RE (2012) Resilience from the point of view of older people:There's still life beyond a funny knee'. Soc Sci Med 74(3):416-424

Wissink B, Schwanen T, Van Kempen R (2016) Beyond residential segregation: Introduction, pp 126-130

Yan X-Y, Zhao C, Fan Y, Di Z, Wang W-X (2014) Universal predictability of mobility patterns in cities. J R Soc Interface 11 : 20140834

Yang J, Leskovec J (2015) Defining and evaluating network communities based on ground-truth. Knowl Inf Syst 42(1):181-213

\section{Publisher's Note}

Springer Nature remains neutral with regard to jurisdictional claims in published maps and institutional affiliations.

\section{Submit your manuscript to a SpringerOpen ${ }^{\circ}$ journal and benefit from:}

- Convenient online submission

Rigorous peer review

- Open access: articles freely available online

High visibility within the field

- Retaining the copyright to your article

Submit your next manuscript at $\boldsymbol{\nabla}$ springeropen.com 